\title{
Vibrational Detection of Hazardous Materials Based on Multivariate Sensors
}

\author{
John R Castro-Suarez*, Ph.D ${ }^{1}$, Samuel P Hernández-Rivera, Ph.D ${ }^{2}$ and Libis C Valdez-Cervantes, M.Sc ${ }^{1}$ \\ ${ }^{1}$ Fundacion Tecnológica Antonio de Arévalo, TECNAR, Colombia, john.castro@tecnar.edu.co, \\ ${ }^{2}$ Universidad de Puerto Rico reciento de Mayagüez, Puerto Rico.
}

\begin{abstract}
A multivariate sensor based on mid-infrared spectral signals have be used for detection of highly hazardous materials (HHMs) employing chemometrics tools. The HHMs used were the nitroaromatic compounds 2,4,6-trinitrotoluene, the aliphatic nitrate ester pentaerythritol tetranitrate and the aliphatic nitramine hexahydrotrinitrotriazine. HHMs were deposited on real-world substrates such as aluminum, cardboard, travel bags and wood. Multivariate analysis by Partial least squares (PLS) regression analysis combined with discriminant analysis (PLS-DA) was used to discriminate, classify, and identity similarities in the spectral datasets. The results show that the Multivariate vibrational detection investigated herein for multivariate sensor development is useful for the detection of HHMs on the types of real-world surface studied.
\end{abstract}

Keywords-multivariate sensors, Chemometrics HHMs, Spectroscopy IR.

Digital Object Identifier (DOI):

http://dx.doi.org/10.18687/LACCEI2016.1.1.331

ISBN: 978-0-9822896-9-3

ISSN: 2414-6390

$14^{\text {th }}$ LACCEI International Multi-Conference for Engineering, Education, and Technology: "Engineering Innovations for Global Sustainability", 20-22 July 2016, San José, Costa Rica. 


\title{
Vibrational Detection of Hazardous Materials Based on Multivariate Sensors
}

\author{
John R Castro-Suarez*, Ph.D ${ }^{1}$, Samuel P Hernández-Rivera, Ph. ${ }^{2}$ and Libis C Valdez-Cervantes, M.Sc ${ }^{1}$ \\ ${ }^{1}$ Fundacion Tecnológica Antonio de Arévalo, TECNAR, Colombia, john.castro@tecnar.edu.co, \\ ${ }^{2}$ Universidad de Puerto Rico reciento de Mayagüez, Puerto Rico. \\ *Corresponding author
}

\begin{abstract}
A multivariate sensor based on mid-infrared spectral signals have be used for detection of highly hazardous materials (HHMs) employing chemometrics tools. The HHMs used were the nitroaromatic compounds 2,4,6-trinitrotoluene, the aliphatic nitrate ester pentaerythritol tetranitrate and the aliphatic nitramine hexahydrotrinitrotriazine. HHMs were deposited on realworld substrates such as aluminum, cardboard, travel bags and wood. Multivariate analysis by Partial least squares (PLS) regression analysis combined with discriminant analysis (PLS-DA) was used to discriminate, classify, and identity similarities in the spectral datasets. The results show that the Multivariate vibrational detection investigated herein for multivariate sensor development is useful for the detection of HHMs on the types of real-world surface studied.
\end{abstract}

Keywords-multivariate sensors, Chemometrics HHMs, Spectroscopy IR.

\section{INTRODUCTION}

Modern society faces an ever increasing need for rapid methods and instrumentation for detection and identification of chemical and biological threat agents. From security antiterrorist personnel, to first responders and law enforcement employees, such as forensic science, police officers, airport screeners, and border patrol personnel, to the Navy, Army, Air Force, and National Guard workforces, the threat of coming in contact with explosive agents is highly pervasive.

Defense and security agencies are in constant demand of new ways of detecting chemical and biological threats used by terrorist organizations. Fundamental and applied research in areas of interest to national defense and security focus in detection of highly energetic materials (HEM) or highly Hazardous materials (HHM) and homemade explosives (HME) that could be used as weapons of mass destruction [13]. Current detection methods of HHM are based on a wide variety of technologies that focus on either bulk amounts or traces of HHM. Bulk explosives can be detected indirectly by imaging characteristic shapes of the explosive charge, detonators, and wires or directly by detecting the chemical composition or dielectric properties of the explosive material. Trace detection methods rely on detection of vapors emitted from the explosives or on explosive particles that are deposited on nearby surfaces [4]. Although there are hundreds of publications about methods of detection of HHM in water, soil, air, clothing, surfaces, etc. and these offer the advantage of providing very low limits of detection (at ppb levels) [5-9], they require, in the majority of the cases, sampling at the scene followed by a sample preparation step, to be later analyzed by a particular technique. Thus, sampling and sample preparation are the main disadvantages in HHM detection, in many cases threatening the health and life of the analyst and first responders. Vibrational spectroscopy, in its various modalities, has shown to be useful for detection of dangerous chemicals, among them HHM and HME.

IR vibrational spectra can to be used for identify and quantify samples in complex matrices because each substance has its own fingerprint spectrum in the mid IR (MIR). This means that IR spectroscopy can be used for discriminant analysis even when the target analyte is in very small quantities [10-12].

Important contributions to the development application doing use of mid infrared vibrational infrared are briefly discussed. Suter et al. studied the spectral and angular dependence of scattered MIR light from surfaces coated with explosives residues (TNT, RDX, and Tetryl) at a $2 \mathrm{~m}$ distance [13]. An external cavity quantum cascade laser provided tunable illumination between 1250 and $1428 \mathrm{~cm}^{-1}$ was used. Kumar and collaborators measured the diffuse reflection spectrum of solid samples such as explosives (TNT, RDX, PETN), fertilizers (ammonium nitrate, urea), and paints (automotive and military grade) at a distance of $5 \mathrm{~m}$ using a mid-infrared supercontinuum light source with $3.9 \mathrm{~W}$ average output power [14]. Kim et al. [15] recently reported that nanomechanical IR spectroscopy provides high selectivity for the detection of TNT, RDX, and PETN without the use of chemoselective interfaces by measuring the photothermal effect of the adsorbed molecules on a thermally sensitive microcantilever.

This contribution aimed at using a ruggedized Midinfrared spectroscopy based explosive detection system that allowed the detection and identification of HHM traces deposited on three types of substrates: travel baggage (TB), cardboard $(\mathrm{CB})$, and wood. Multivariate models were developed from spectroscopy data to get multivariate sensors that allow to detect y classification de HEM on real surfaces. One chemometrics routines was applied to analyze the characteristics of the recorded MIRS spectra: partial least squares (PLS) regression analysis coupled with discriminant analysis (PLS-DA), which was used to discriminate, classify,

Digital Object Identifier (DOI): http://dx.doi.org/10.18687/LACCEI2016.1.1.331 ISBN: 978-0-9822896-9-3

ISSN: $2414-6390$

$14^{\text {th }}$ LACCEI International Multi-Conference for Engineering, Education, and Technology: "Engineering Innovations for Global Sustainability”, 20-22 July 2016, San José, Costa Rica. 
and identity similarities among the spectral data. Several preprocessing steps were applied prior to the multivariate analysis protocols employed. The results indicate that the MIRS-based methodology described in this study can be used for rapid screening analysis of HEMs.

\section{MATERIALS AND METHODS}

\section{A. Reagents.}

Reagents used in this investigation included highly hazardous materials and solvents. 2,4,6-trinitrotoluene (TNT) was acquired from ChemService, Inc. (West Chester, PA) as crystalline solids $(99 \%$, min. 30\% water content). 1,3,5trinitroperhydro-1,3,5-triazine or hexahydrotrinitrotriazine (RDX) and pentaerythritol tetranitrate (PETN) were synthesized at micro scale in the lab. Methanol (99.9\%, HPLC grade), dichloromethane $\left(\mathrm{CH}_{2} \mathrm{Cl}_{2}, \mathrm{HPLC}\right.$ grade) and acetone (99.5\%, GC grade) were purchased from Aldrich-Sigma Chemical Co. (Milwaukee, WI) and were used to deposit the HHM samples with different surface concentrations onto TB, $\mathrm{CB}$, and wood used as substrates.

\section{B. Instrumentation.}

Detection of PETN, RDX and TNT deposited on real substrates was carried out using a LaserScan ${ }^{\mathrm{TM}}$ acquired from Block Engineering, LLC (Marlborough, MA). This instrument uses a MIR widely tunable QCL. The sensitivities that can be achieved using this spectroscopic system are much higher than those achieved with ordinary dispersive IR systems equipped with thermal excitation sources. MIR spectra were recorded in the spectral range of $1000-1600 \mathrm{~cm}^{-1}$ at a distance of 6 in. All spectra were taken at 2 co-adds and $4 \mathrm{~cm}^{-1}$ resolution. Fig. 1 shows the experimental setup used in this research for carried out sensing and development multivariate sensor.

\section{Sample preparation}

Detection of solid samples present as traces on substrates (surfaces) required a sample preparation methodology that would be able to deposit solid samples on a solid substrate, with high coverage uniformity and reproducibility. Due to the size of the substrates, sample smearing and partial immersion technique was used to deposit the solid analytes at trace amounts on the metallic and no-metallic substrates. Substrates were aluminium (AL), cardboard (CB), travel bags (TB) and wood. Metallic and no-metallic substrates of areas $5.0 \mathrm{~cm} \times$ $5.0 \mathrm{~cm}$ were used as material support for solid samples. Methanol was used to clean the surfaces tested. Substrates were allowed to air-dry before of depositing the desired HHM surface loading. A small amount of dichloromethane was used to dissolve target sample to be deposited. The nominal surface concentrations obtained by the deposition technique used were between: $1-20 \mu \mathrm{g} / \mathrm{cm}^{2}$. A total of 460 independent measurements (spectrum) were taken for three type HHM and three different substrates.

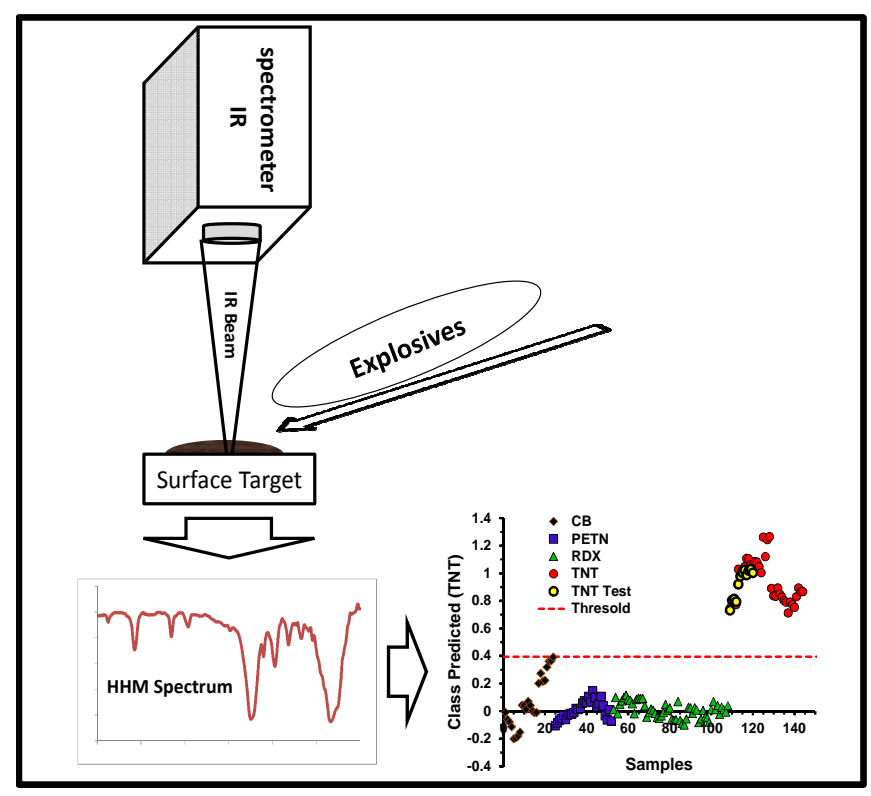

Fig 1 Experimental setup for HHM detection on real-world substrates

\section{RESULTS}

A. Vibrational detection.

Mid infrared vibrational spectra of RDX, TNT and PETN, deposited on TB, CB and wood substrates were acquired using QCL spectrometer. Four spectra were obtained for each substrate sample. The spectra were acquired using as background the substrate without HHM deposited on them. Spectra were record in the MIR spectral region of 1000$1600 \mathrm{~cm}^{-1}$ where the characteristic vibrations of the HHM occur. Fig. 2-3 shows some IR vibrational spectra of HHM deposited on different substrate tested.

The most import vibrational bands that can be observed in Fig. 2 are to TNT were $1024 \mathrm{~cm}^{-1}, 1086 \mathrm{~cm}^{-1}, 1350 \mathrm{~cm}^{-1}$, and $1551 \mathrm{~cm}^{-1}$ [16]. For PETN, some of the important signatures appeared at $1003 \mathrm{~cm}^{-1}, 1038 \mathrm{~cm}^{-1}, 1272 \mathrm{~cm}^{-1}, 1285 \mathrm{~cm}^{-1}$, and $1306 \mathrm{~cm}^{-1}$ [17]. Finally, important bands for RDX were detected at $997 \mathrm{~cm}^{-1}, 1220 \mathrm{~cm}^{-1}, 1270 \mathrm{~cm}^{-1}, 1310 \mathrm{~cm}^{-1}, 1420$ and $1445 \mathrm{~cm}^{-1}$, and $1570 \mathrm{~cm}^{-1}[18]$.

In contrast, when the HHM spectra were acquired from non-metallic substrates (see Fig. 3), the reflection component of the transflection spectra stands out more prominently, producing reflection spectra with profiles similar those with anomalous dispersions [19]. These spectral features are usually observed when the sample refractive index decreases to the high-wavenumber side of the absorption band maximum, returning to the normal value at the absorption band center and then increasing to the lower wavenumber side before returning again to the normal values [20]. However this spectral distortions observed are not anomalies but, rather, spectral profiles dominated by the reflection of the HHM samples [21]. 

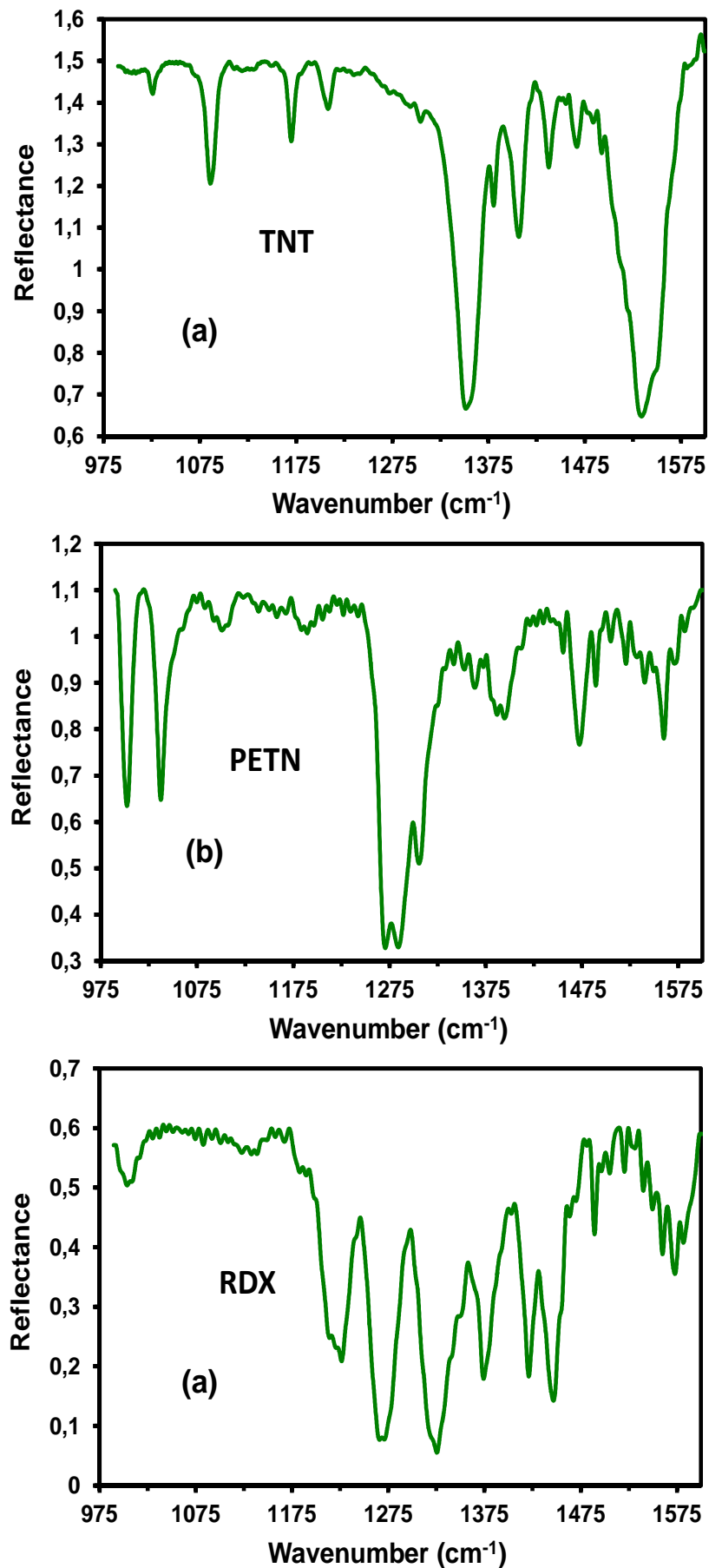

Fig. 2 Vibrational spectra of HHM, (a) TNT, (b) PETN and (c) RDX on Aluminium.

\section{B. Development of Multivariate Sensor}

For analyze the IR vibrational data recorded, PLS Toolbox ${ }^{\mathrm{TM}}$ version 6.5 (Eigenvector Research Inc., Wenatchee, WA, USA) for MATLAB ${ }^{\mathrm{TM}}$ (The MathWorks, Inc. Natick, MA, USA) was used. All the data recorded was divided into two groups: a calibration set and a prediction set. The calibration set contained approximately $70 \%$ of the data and the prediction set contained approximately $30 \%$ of the data. PLSDA was applied to the spectral data in order to classify or group all spectra by HHM type and to discriminate between clean substrate contributions in the MIR and substrates with explosives. PLS-DA is one of the most widely used chemometrics tools, particularly when the goal is to discriminate, classify and identity spectral similarities in a multivariate data set. PLS-DA is a supervised pattern recognition method. A detailed explanation of how PLS-DA works and its numerous applications is not included in this paper. Excellent in-depth mathematical support and various applications in natural sciences and engineering are available in the literature [22-24]. PLS-DA is a linear classification method that combines the properties of PLS regression with the discrimination power of a classification technique. PLSDA is based on the PLS regression algorithm, which searches for latent variables with a maximum covariance between a descriptor matrix $\mathbf{X}$ and a response matrix $\mathbf{Y}$ (containing the membership of samples to the $G$ classes expressed with a binary code: 1 or 0 ). The primary advantage of PLS-DA is that the relevant sources of data variability are modeled by the socalled latent variables (LVs), which are linear combinations of the original variables, and consequently, allowing graphical visualization and understanding of the different data patterns and relations by LV scores and loadings. The scores represent the coordinates of the samples in the LV projection hyperspace $[23,24]$. Several preprocessing steps were applied to the data with the objective of generating multivariate models capable of clustering spectra by chemical similarities i.e. PETN, RDX, TNT and clean substrates (TB, CB and wood).

The fig. 4 and 5 show the score plots resulting from the PLS-DA runs. The score plots allow visualization of the clustering of the spectral data and demonstrate that the best results were obtained for the models generated after preprocessing was applied. The class predictions of PETN, RDX, and TNT on Wood from the cross-validation set are shown in Fig. 4a-4c. Five Latent Variables (LVs) were required to obtain the best multivariate classification model.

For the multivariate analysis of the mid-IR vibrations of the HHMs deposited on wood, it was necessary to preprocess the data by taking the first derivative (15 points) and using SNV transformation and Mean Centering (MC) to obtain the best chemometrics results.

For the multivariate analysis of the mid-IR vibrations of the HHMs deposited on TB and $\mathrm{CB}$, we obtained the best chemometrics models by taking the first derivative (15 points) and applying MC. 

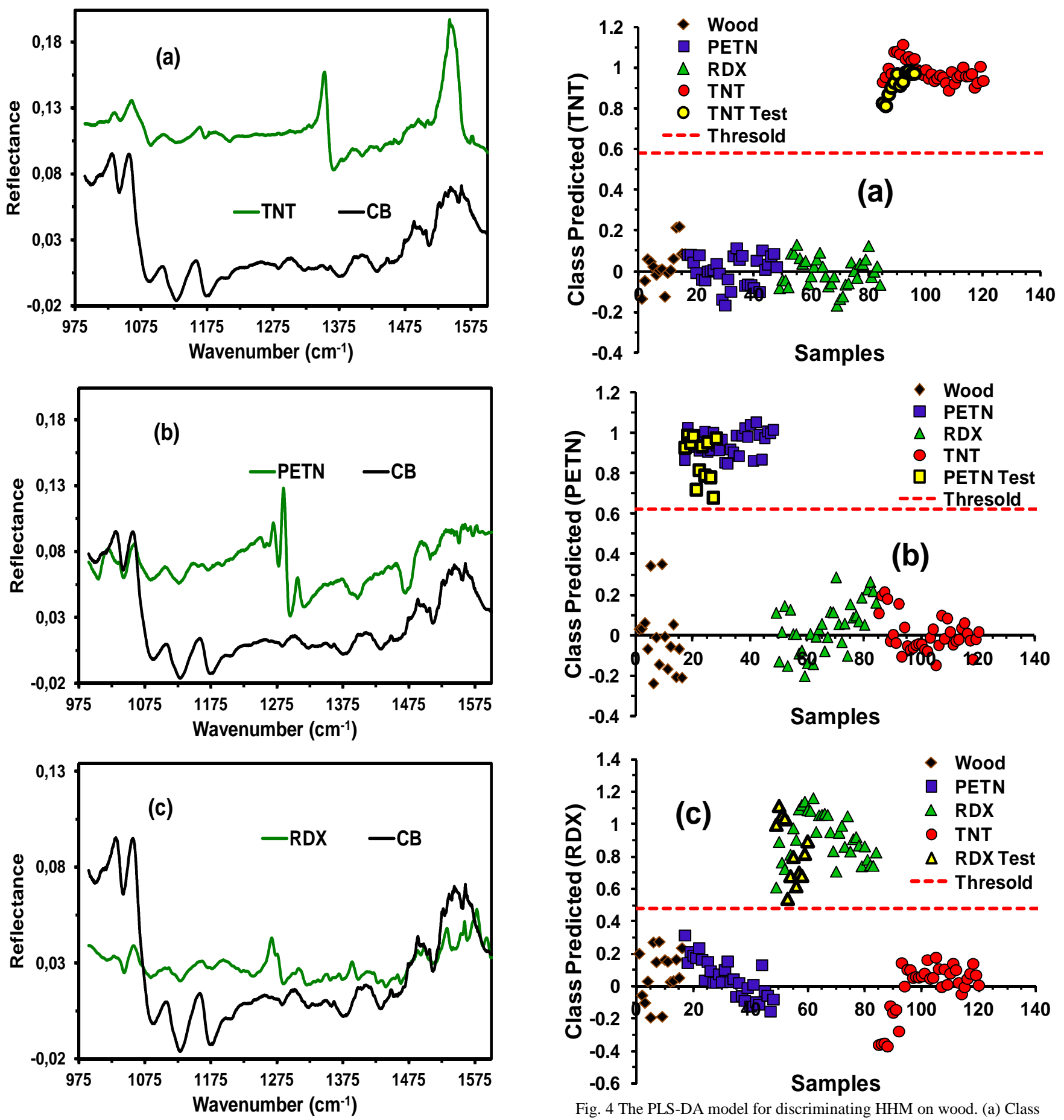

Fig. 4 The PLS-DA model for discriminating HHM on wood. (a) Class prediction for TNT. (b) Class prediction for PETN. (c) Class prediction for Cardboard-CB RDX.

$14^{\text {th }}$ LACCEI International Multi-Conference for Engineering, Education, and Technology: "Engineering Innovations for Global Sustainability", 20-22 July 2016, San José, Costa Rica. 


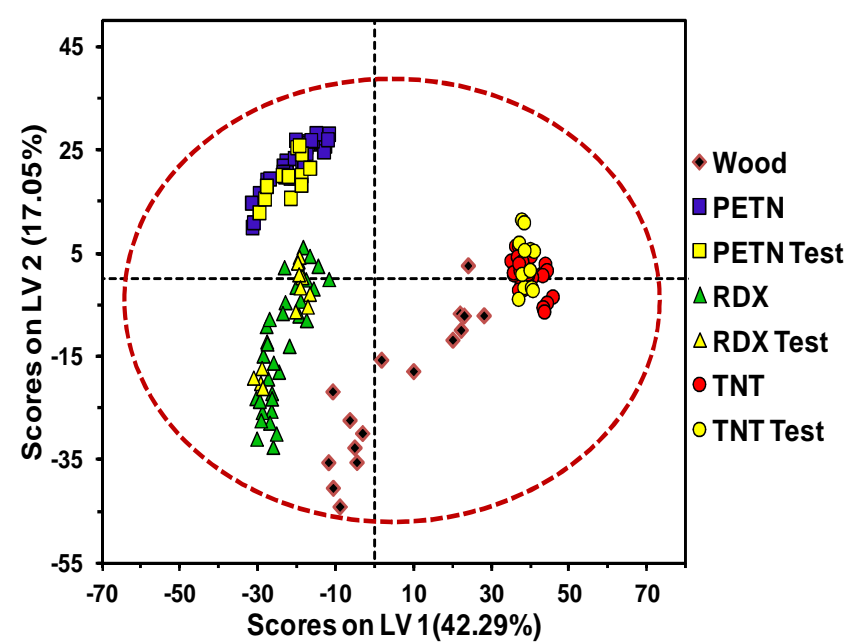

Fig. 5 Score plots of LV2 versus LV1 for the detection of PETN, RDX, and TNT on Wood

\section{CONCLUSION}

Mid infrared spectroscopy system was used for the detection of HHMs deposited at low surface concentrations on three types of non-ideal substrates: polyester travel-bag fabric (TB), cardboard (CB), and wood. The spectral profiles of HHMs in transflection experiments depend on the reflectivity of the substrate. For highly reflective substrates, such as $\mathrm{Al}$, the transflection spectra are similar to transmission spectra; for low-reflectivity substrates, such as $\mathrm{TB}, \mathrm{CB}$, and wood, the transflection spectra are similar to reflection spectra, where nitro group bands prevail. For the multivariate analysis on TB and $\mathrm{CB}$ preprocessing as $1^{\text {st }}$ derivative and mean center were sufficient to develop multivariate sensor. In Wood substrate by to be surface more complex a third preprocessing (SNV) was necessary. In general, we have demonstrated that MIRS is useful for developing a rapid screening methodology for the detection and discrimination of HEMs on nonideal, lowreflectivity, highly interfering substrates using multivariate analysis.

\section{REFERENCES}

[1] M. Marshall, and J. C. Oxley, "Aspects of Explosives Detection", Elsevier, Amsterdam, The Netherlands, 2009.

[2] J. Yinon, and S. Zitrin, "Modern Methods and applications in analysis of explosives", John Wiley \& Sons Ltd., Chichester, UK, 1996.

[3] H. Schubert, and A. Rimski-Korsakov, "Stand-Off Detection of SuicideBombers and Mobile Subjects", Proceedings of the NATO Advanced Research Workshop on Stand-Off Detection of Suicide Bombers and Mobile Subjects, NATO Security through Science Series B: Physics and Biophysics, Pfinztal, Germany, Springer, Germany, 2005.

[4] Committee on the Review of Existing and Potential Standoff Explosives Detection Techniques, "Existing and Potential Standoff Explosives Detection Techniques", National Research Council, National Academy of Sciences Committee, Washington, D.C, 2004.

[5] T. Caron, M. Guillemot, P. Montméat, F. Veignal, F. Perraut, P. Prené, and F. Serein-Spirau, "Ultra trace detection of explosives in air:
Development of a portable fluorescent detector," Talanta, vol. 81, pp. 543-548, 2010.

[6] Hilmi and J. Luong, "Micromachined Electrophoresis Chips with Electrochemical Detectors for Analysis of Explosive Compounds in Soil and Groundwater," Environ. Sci. Technol., vol. 34, pp. 3046-3050, 2000.

[7] J. Yinon, "Trace analysis of explosives in water by gas chromatography-mass spectrometry with a temperatureprogrammed injector," J. Chrom. A, vol. 742, pp. 205-209, 1996.

[8] C. Szakal and T. M. Brewer, "Analysis and Mechanisms of Cyclotrimethylenetrinitramine Ion Formation in Desorption Electrospray Ionization," Anal. Chem., vol. 81, pp. 5257-5266, 2009.

[9] C. J. Miller and T. S. Yoder, "Explosive Contamination from Substrate Surfaces: Differences and Similarities in Contamination Techniques Using RDX and C-4," Sens. Imaging: An International Journal. vol .11, pp. 77-87. 2010.

[10] H. Gunzler and H.-U. Gremlich, "IR Spectroscopy: An Introduction," Wiley-VCH, Weinheim, Germany, 2002.

[11] S. Bangalore, G. W. Small, R. J. Combs, R. B. Knapp, R. T. Kroutil, C. A. Traynor, and J. D. Ko, "Automated Detection of Trichloroethylene by Fourier Transform Infrared Remote Sensing Measurements," Anal. Chem. vol. 69, pp. 118-129, 1997.

[12] Smith, "Fundamentals of Fourier Transform Infrared Spectroscopy," CRC Press, Boca Raton, FL, USA, 2000.

[13] J. D. Suter, B. Bernacki and M.C. Phillips, "Spectral and angular dependence of mid-infrared diffuse scattering from explosives residues for standoff detection using external cavity quantum cascade lasers," Applied Physics B, vol. 108, no. 4, pp. 965-974, 2012.

[14] M. Kumar, M. N. Islam, F.L. Terry, M.J. Freeman, A. Chan, M. Neelakandan and T. Manzur, "Stand-off detection of solid targets with diffuse reflection spectroscopy using a high-power mid-infrared supercontinuum source,"' Applied optics, vol. 51, no. 15, pp. 2794-2807, 2012.

[15] S. Kim, D. Lee, X. Liu, C. Van Neste, S. Jeon and T. Thundat, "Molecular Recognition Using Receptor-Free Nanomechanical Infrared Spectroscopy Based on a Quantum Cascade Laser," Scientific reports, vol. 3, Article number 1111, 2013.

[16] J. Clarkson, W.E. Smith, D.N. Batchelder, D.A. Smith, and A.M. Coats, "A Theoretical Study of the Structure and Vibrations of 2,4,6Trinitrotolune,'” J. Mol. Struct.vol. 648, no.3, pp. 203-214, 2003.

[17] W.F. Perger, J. Zhao, J.M. Winey, and Y.M. Gupta, "First-Principles Study of Pentaerythritol Tetranitrate Single Crystals Under High Pressure: Vibrational Properties,' Chem. Phys. Lett., vol. 428, no. 4-6, pp. 394399. 2006.

[18] R.L. Prasad, R. Prasad, G.C. Bhar, and S.N. Thakur, "Photoacoustic Spectra and Modes of Vibration of TNT and RDX at CO2 Laser Wavelengths,' Spectrochim. Acta, Part A, vol. 58, no. 14, pp. 30933102, 2002.

[19] D.M. Hembree, and H.R. Smyrl, “Anomalous Dispersion Effects in Diffuse Reflectance Infrared Fourier Transform Spectroscopy: A Study of Optical Geometries," Appl. Spectrosc., vol. 43, no. 2, pp. 267-274. 1989.

[20] J.M. Chalmers, "Mid-Infrared Spectroscopy: Anomalies, Artifacts and Common Errors" In Handbook of Vibrational Spectroscopy, J.M. Chalmers, and P.R. Griffiths, Chichester, UK: John Wiley and Sons, Vol. III, p p. 2327-2347, 2006.

[21] P. Bassan, H.J. Byrne, J. Lee, F. Bonnier, C. Clarke, P. Dumas, E. Gazi, M.D. Brown, N.W. Clarke, and P. Gardner, "Reflection Contributions to the Dispersion Artefact in FTIR Spectra of Single Biological Cells," Analyst, vol. 134, no. 6, p p. 1171-1175, 2009.

[22] M. Barker, and W. Rayens, "Partial Least Squares for Discrimination," J. Chemom., vol. 17, no. 3, pp. 166-173, 2003.

[23] R.G. Brereton, "Chemometrics for Pattern Recognition". Chichester, England. The Atrium, Southern Gate: John Wiley \& Sons Ltd. 2009.

[24] D. Ballabio, V. Consonni, "Classification Tools in Chemistry. Part 1: Linear Models. PLS-DA,” Anal. Methods., vol. 5, no. 16, pp. 3790-3798, 2013. 